\title{
Detecting Density Dependence in Recovering Seal Population is Difficult: A Response to Svensson et al. (2011)
}

\author{
Mike Lonergan
}

Received: 7 April 2011/Revised: 2 July 2011/Accepted: 5 July 2011 / Published online: 9 September 2011

This comment was peer reviewed.

Comment to: Svensson, C. J., A. Eriksson, T. Harkonen and K. C. Harding. 2011. Detecting density dependence in recovering seal populations. AMBIO 40:52-59.

A recent simulation study (Svensson et al. 2011) proposed the use of changes in age-structure as an indicator of density dependent effects on the growth of the Baltic grey seal (Halichoerus grypus) population. That approach provides a clever alternative to the direct examination of abundance estimates, and sidesteps the well-known difficulty of detecting trends in marine mammal populations from survey data (Taylor et al. 2007). However, there are three features of Svensson et al.'s analysis that limit its utility as a demonstration of the practicality of their methodology as a tool for investigating real-world problems.

\section{ARTEFACTS}

The first issue is that the results, as presented, seem to contain artefacts, patterns produced by the analysis rather than arising from the data. Simulations where the environmental carrying capacity was quite stable and density dependence only acted on populations close to their carrying capacity contained multiple periods when density dependence was detectable (Svensson et al. 2011, Figs. 3c, $4 c)$. The initial periods were the intuitively obvious ones: late on in the growth of the population while it was

The online version of the article commented upon can be found at doi:10.1007/s13280-010-0091-7 decelerating towards the carrying capacity. The others seem to be associated with an overcompensatory response in the model (Svensson et al. 2011, Fig. 2b), a feature those authors noted. Such effects are sensitive to many factors including: the pattern of density dependent mortality; whether it exclusively affects pups; and the distribution of ages of first reproduction of females. Changes in any of these could alter the timing, duration and number of periods in which density dependent effects are detectable. None of them are easy to investigate, and the prevalence of overcompensatory effects in actual pinniped population trajectories seems an unexplored area. The examples in Svensson's paper suggest that density dependence is detectable for longer periods when abundance, rather than age-structure, is used as the indicator (Svensson et al. 2011, Figs. 3, 4), but it is difficult to anticipate how the relative durations would be affected by modifications to the model's structure.

\section{METHOD FOR DETECTING DENSITY DEPENDENCE}

A second difficulty arises out of a mismatch between the population model and the regression used to identify density dependence. The population model appears almost deterministic, with stochasticity only occurring in the environmental carrying capacity. No uncertainty was described in the assessment of abundance. A significant quadratic term in a regression of $\log$ (abundance) against time was used to indicate the occurrence of density dependent effects. Regression assumes that each datapoint is independently drawn from a distribution centred on the appropriate point on an overall trendline (Gerrodette 1987), but these data are not independent: each year the abundance lies below the 
trend is likely to be followed by another one. This could lead the regression to incorrectly report a significant trend. The regression method is appropriate for data from real surveys, where environmental effects on haulout behaviour dominate the variation in annual growth rates (Thompson et al. 2005). The problem is slightly different in Svensson's analysis of the changes in population age-structure: the sampling process does introduce stochasticity, but this follows a binomial rather than the assumed normal distribution. Recasting the model so that the response data is the ratio of consecutive counts, and the explanatory variable is the numbers counted in the first of each pair of years, might allow regression to be used for this analysis, though the autocorrelation in annual population growth rates introduced by varying age-structures (Härkönen et al. 2002) could remain problematic.

\section{REPRESENTATION OF UNCERTAINTY}

The third, and most important, issue is the representation of uncertainty in the analysis. It is not immediately obvious that uncorrelated annual variation in the environmental carrying capacity is the main stochastic effect on the population. While the error in abundance estimates limits their use to detect trends (Taylor et al. 2007), behavioural differences relating to animals' sex and age are more problematic: these make it hard both to know how to select a representative sample of grey seals, and to check that a particular sample is sufficiently representative. Svensson's age-structure method relies on aging a representative sample of around 1000 individuals each year. The binomial sampling error assumes each individual is effectively chosen independently. Determining the ages of 1000 live seals is not a trivial undertaking: methods involving handling each one seem infeasible. Photography might allow the estimation of length, mass and secondary sexual characteristics, but cannot always unequivocally indicate maturity. Biases that vary between years could indicate spurious patterns or conceal real changes. Svensson et al. do mention the existence of difficulties associated with observation error, but do not incorporate its effects into their analysis.

\section{CONCLUSION}

The approach Svensson et al. suggest is certainly interesting. However, in its current form, their analysis seems likely to underestimate the difficulty of using changes in age-structure to detect the impact of density dependence on the recovery of the Baltic grey seal population. Further work will be needed to examine more fully the uncertainties associated with real data. If that identifies conditions under which Svensson's age-structure method outperforms the direct examination of abundance estimates, its adoption might reduce the effort involved in the detection of changing population trajectories, and free scarce resources for other investigations.

Acknowledgments The author is grateful to the anonymous reviewers of this Comment. They corrected some of his misapprehensions.

\section{REFERENCES}

Gerrodette, T. 1987. A power analysis for detecting trends. Ecology 68: 1364-1372.

Härkönen, T., K.C. Harding, and M.P. Heide-Jorgensen. 2002. Rates of increase in age-structured populations: a lesson from the European harbour seals. Canadian Journal of Zoology (Revue Canadienne De Zoologie) 80: 1498-1510.

Svensson, C.J., A. Eriksson, T. Harkonen, and K.C. Harding. 2011. Detecting density dependence in recovering seal populations. AMBIO 40: 52-59.

Taylor, B.L., M. Martinez, T. Gerrodette, J. Barlow, and Y.N. Hrovat. 2007. Lessons from monitoring trends in abundance of marine mammals. Marine Mammal Science 23: 157-175.

Thompson, D., M. Lonergan, and C. Duck. 2005. Population dynamics of harbour seals Phoca vitulina in England: monitoring growth and catastrophic declines. Journal of Applied Ecology 42: 638-648.

Mike Lonergan ( $\square)$

Address: NERC Sea Mammal Research Unit, Scottish Oceans Institute, University of St Andrews, St Andrews KY16 8LB, UK.

e-mail:mel5@st-and.ac.uk 